\title{
Close concordance between pulmonary angiography and pathology in a canine model with chronic pulmonary thromboembolism and pathological mechanisms after lung ischemia reperfusion injury
}

\author{
Chaosheng Deng ${ }^{1} \cdot$ Dawen $\mathrm{Wu}^{1} \cdot$ Zhenguo Zhai $^{2} \cdot$ Qichang Lin $^{1} \cdot$ Zhanghua Zhong $^{1} \cdot$ \\ Yuanhua Yang ${ }^{2} \cdot$ Qunlin Chen $^{3} \cdot$ Ningfang Lian $^{1} \cdot$ Shaoyong Gao $^{1}$ • \\ Minxia Yang ${ }^{1} \cdot$ Kaixiong Liu ${ }^{1}$. Chen Wang ${ }^{4}$ \\ Published online: 19 August 2015 \\ (c) The Author(s) 2015. This article is published with open access at Springerlink.com
}

\begin{abstract}
To investigate the pulmonary angiography and pathology in a canine model with chronic pulmonary thromboembolism (PTE). The cylindrical blood clots were selectively introduced into the left $(\mathrm{n}=10)$ or right $(n=20)$ lower pulmonary arteries of dogs. Pulmonary arteriography (PA) was performed before or after embolization. The values after embolization and baseline of mean pulmonary arterial pressure, pulmonary vascular resistance, cardiac output had changed. After 1 or 2 weeks' embolization, local PA demonstrated the abrupt cut-off perfusion defects or webs, bands, and abrupt vascular narrowing. 2 weeks after embolization, the pathology showed that the fibrin networks of the thrombi had multiple recanalization channels, and pulmonary artery had the concentric, lamellar (onion-like) intimal hyperplasia, multilayered, irregular arrangements of endothelial cells, and the infiltration of inflammatory cells. After embolectomymediated reperfusion, 2 weeks' subgroup showed destroyed and incomplete alveolar structures, and a large number of exudative cells, primarily neutrophils, and exudate. There close concordance between pulmonary
\end{abstract}

Chaosheng Deng

jasonsci7333@163.com

1 Division of Respiratory and Critical Care Medicine, First Affiliated Hospital of Fujian Medical University, Fuzhou 350005, Fujian, China

2 Division of Respiratory and Critical Care Medicine, Beijing Institution of Respiratory Medicine, Beijing Chaoyang Hospital, Capital Medical University, Beijing 100020, China

3 Department of Medical Imaging, First Affiliated Hospital of Fujian Medical University, Fuzhou 350005, Fujian, China

4 Beijing Key Laboratory of Respiratory and Pulmonary Circulation, Institute of Respiratory Medicine, Beijing Hospital, Ministry of Health, Beijing 100730, China angiography and pathology in a canine model with chronic PTE. The LIRI mechanisms after embolectomy-mediated reperfusion involve the destroyed, incomplete alveolar structures, and infiltration of inflammatory cells, primarily neutrophils.

Keywords Pulmonary thromboembolism $\cdot$ Pulmonary angiography $\cdot$ Lung ischemia reperfusion injury $\cdot$ Animal model $\cdot$ Pathology

$\begin{array}{ll}\text { Abbreviations } \\ \text { PTEs } & \text { Pulmonary thromboembolisms } \\ \text { DVT } & \text { Deep vein thrombosis } \\ \text { VTEs } & \text { Venous thromboembolisms } \\ \text { PEs } & \text { Pulmonary embolisms } \\ \text { TXA } & \text { Tranexamic acid } \\ \text { LIRI } & \text { Lung ischemia reperfusion injury } \\ \text { CTEPH } & \text { Chronic thromboembolic pulmonary } \\ & \text { hypertension } \\ \text { PVC } & \text { Polyvinyl chloride } \\ \text { RR } & \text { Respiratory rate } \\ \text { HR } & \text { Heart rate } \\ \text { MBP } & \text { Mean blood pressure } \\ \text { PaO } & \text { Oxygen partial pressure } \\ \text { PaCO } & \text { Carbon dioxide partial pressure } \\ \text { CVP } & \text { Central venous pressure } \\ \text { MPAP } & \text { Mean pulmonary arterial pressure } \\ \text { PAWP } & \text { Pulmonary artery wedge pressure } \\ \text { CO } & \text { Cardiac output } \\ \text { PVR } & \text { Pulmonary vascular resistance } \\ \text { PA } & \text { Pulmonary angiography } \\ \text { HE } & \text { Hematoxylin and eosin } \\ \text { PTAH } & \text { Phosphotungstic acid-hematoxylin } \\ \text { EC } & \text { Endothelial cell } \\ \text { MCP-1 } & \text { Macrophage chemoattractant protein-1 } \\ & \end{array}$




\section{Introduction}

Pulmonary thromboembolisms (PTEs) are currently the third most common cause of death among hospitalized patients [1]. Animal models of PTE have helped enhance our understanding of the pathogenesis and pathophysiological changes of this syndrome, have suggested methods for diagnosis, and provided a means for evaluating new pharmaceutical-based prophylactic and therapeutic approaches [2]. In the broadest terms, these models can be categorized as being induced by using an injected thrombus or a foreign body. The injected clot model provides a closer representation of PTEs; however, the extent and persistence of the resultant pulmonary vessel occlusion can be difficult to control because of the target animal's remarkably efficient fibrinolytic system [3, 4]. To counter the effects of the fibrinolytic system, tranexamic acid (TXA) an inhibitor of plasmin — can be used to inhibit endogenous fibrinolysis in animals $[4,5]$. The irregular sizes and volumes of ex vivo-produced clots allow them to flow freely into different pulmonary arteries. Moreover, the pathological results show that pulmonary emboli released from peripheral veins or the vena cava can be impeded by the right heart valves, papillary muscle and chordae tendineae (Fig. 1: 1), as often observed in clinical cases [5, 6]. All these aspects influence the effects of PTE on hemodynamics, cardiac function, and other such parameters [7]. Therefore, experimental models that more accurately reflect the predicted in vivo effects of an intervention would facilitate the assessment and comparison of different antithrombotic agents, and their efficacy and long-term outcomes in PTE treatment [8]. Moreover, lung ischemia reperfusion injury (LIRI) may occur in the region of the affected lung, after thrombolytic therapy, pulmonary embolectomy, or thrombarterectomy in patients with chronic thromboembolic pulmonary hypertension (CTEPH) [9] [10]. The mechanisms of LIRI in PTE are indistinct because of the difficulty in establishing an appropriate ischemia-reperfusion model of PTE.

The canine genome exhibits greater fibrinolytic system homology with the human genome as compared to other mammalian models of thrombotic disease [11, 12]. Interestingly, Virchow's original description of a canine model focused on the development of animal models of thrombosis that mimicked the human condition [13]. Therefore, in the present study, we aimed to establish a modified canine PTE model involving blood clots that were selectively introduced into the intended specific pulmonary lobar artery and explore the probable pathological and cellular mechanisms of LIRI after the embolectomy.

\section{Materials and methods}

\section{Animals and groups}

The various procedures were approved by the Fujian Medical University Institutional Animal Care and Use committee, and all experiments were conducted in accordance with the Guide for the Care and Use of Laboratory Animals (United States National Institutes of Health, Bethesda, MD). Forty, healthy, 2-year-old dogs (weight, $20 \pm 1.9 \mathrm{~kg}$ ) were divided into 2 groups. The group LL $(\mathrm{n}=10)$ animals had 3-segmented, cylindrical, autologous blood clots introduced into their left main lower pulmonary arteries for 1 week mainly for observing the feasibility of selective embolization. The group RL $(\mathrm{n}=30)$ animals had similar cylindrical blood clots selectively introduced to embolize the right main lower pulmonary arteries for monitoring and comparing vital signs, blood gases, and hemodynamic parameters. The group RL was subdivided into 3 subgroups. The first of these was the Sham subgroup $(\mathrm{n}=10)$. These animals underwent the same procedures as those in the other subgroups, except that $0.9 \% \mathrm{NaCl}$ was infused into the lower pulmonary artery of each animal, instead of blood clots, and the animals were observed for 2 weeks. The 1 -week subgroup $(\mathrm{n}=10)$ received 3 -segmented, cylindrical, autologous blood clots. These clots were introduced into the right lower arteries and the animals were observed for 1 week. The 2-week subgroup $(\mathrm{n}=10)$ underwent the same procedures as the 1-week subgroup, but these animals were observed for 2 weeks. In 5 of the animals in this subgroup, embolectomies were performed for reperfusion, based on the exact location of the thrombus. In the other 5 animals, the lungs were dissected and the lower pulmonary arteries, with the thrombi, were incised for observation.

\section{Establishing a modified experimental LIRI canine model of PTE}

\section{Preparation of the cylindrical autologous blood clots}

Autologous blood $(20 \mathrm{~mL})$ was extracted from the saphenous vein of each dog, using a 20 -mL syringe, and rapidly injected into 3-segmented, sterile intravenous polyvinyl chloride (PVC) tubes (Shanghai Muhe Medical Material, Shanghai, China) at room temperature. The tube sections were $7-\mathrm{cm}$-long, with inner diameters of $4 \mathrm{~mm}$, and were used to form the cylindrical, autologous blood clots. After $8 \mathrm{~h}$, the clots were gently aspirated into another PVC tube (tube I; length, $25 \mathrm{~cm}$, inner diameter, $5 \mathrm{~mm}$ ) for later use (Fig. 1: 2, 3). 

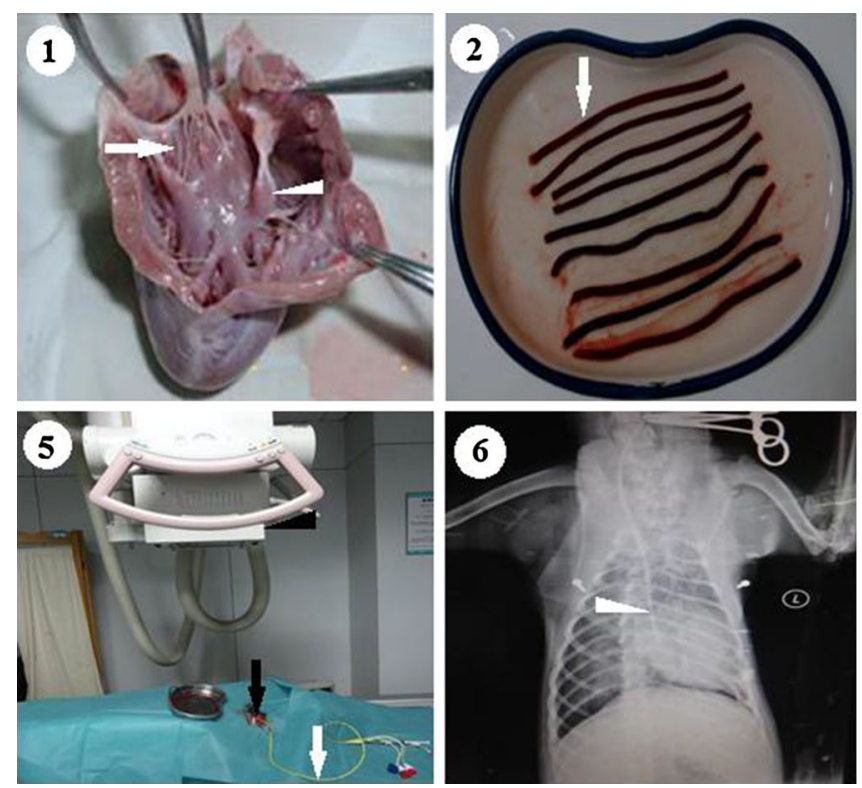

Fig. 1 The procedures of establishing the animal model. The dog's pathological results showed that pulmonary emboli released from peripheral veins can be impeded by the right heart valves, papillary muscle $(\mathbf{1}$, white arrow head) and chordae tendineae $(\mathbf{1}$, white arrow). The cylindrical, autologous blood clots were formed by the tube sections $(\mathbf{2}$, white arrow). After $8 \mathrm{~h}$, the clots were gently aspirated into another PVC tube (tube I) (3, black arrow head). The tube II (3, 4, white arrow head) was guided by the Swan-Ganz float catheter (4, white arrow) to float selectively into the left or right lower pulmonary

\section{Establishing the animal model and pulmonary angiography}

Each dog was anesthetized with intravenous propofol $(2.5 \mathrm{mg} / \mathrm{kg})$ and intraperitoneal $3 \%$ sodium pentobarbital $(0.5 \mathrm{~mL} / \mathrm{kg}$; Changzhou Medical Material, Changzhou City, China), and intubated. Thereafter, each animal received a single bolus dose of intravenous TXA $(110 \mathrm{mg} /$ $\mathrm{kg})$. Vital signs, including respiratory rate (RR), heart rate (HR), and mean blood pressure (MBP), were recorded according to the experimental scheme. Blood pressure was monitored using a right femoral artery cannula (Changzhou Medical Material). Arterial blood $\mathrm{pH}$, oxygen partial pressure $\left(\mathrm{PaO}_{2}\right)$, and carbon dioxide partial pressure $\left(\mathrm{PaCO}_{2}\right)$ were also periodically monitored. A Swan-Ganz float catheter (Edwards Lifesciences, Irvine, CA, USA) was used to guide a $40-\mathrm{cm}$ PVC tube (inner diameter, $5 \mathrm{~mm}$; tube II) to float selectively into the left or right lower pulmonary artery, under fluoroscopic guidance (Fig. 1: 4). The right external jugular vein was dissected and cannulated with a 7-Fr sheath (Fig. 1: 5) (Shanghai Muhe Medical Material). The Swan-Ganz catheter was connected to a pressure transducer and a multi-channel signal analysis system (Shanghai Alcott Biotech, Shanghai City, China) to monitor the central venous pressure (CVP),



artery under fluoroscopy $(\mathbf{5}, \mathbf{6})$. The tube I and II were connected with another, larger PVC tube (3, white arrow). Digital subtraction pulmonary angiography (PA) was performed before embolization and showed no filling defects within both pulmonary artery $\mathbf{7}$, white arrow). The blood clots prepared inside tube I $(\mathbf{8}$, black arrow head $)$ were infused into the lower pulmonary artery through tube II $(\mathbf{8}$, white arrow head $)$ by the dissected right external jugular vein $(\mathbf{8}$, black arrow)

mean pulmonary arterial pressure (MPAP), and pulmonary artery wedge pressure (PAWP); measure the cardiac output (CO) using the thermal dilution method; and calculate pulmonary vascular resistance $(\mathrm{PVR}=[(\mathrm{MPAP}-$ PAWP $/ \mathrm{CO}] \times 80$ ). Digital subtraction pulmonary angiography (PA) (GE Healthcare, Little Chalfont, Buckinghamshire, UK) was performed using Ultravist (300 mg/ mL, Seling Pharmaceutical, Guzhou, China, Fig. 1: 6, 7). After guiding tube II into the left or right lower artery, the Swan-Ganz catheter was extracted from inside tube II. Tubes I and II were connected with another, larger PVC tube (length, $3 \mathrm{~cm}$; inner diameter, $6 \mathrm{~mm}$ ) (Fig. 1: 3), and the blood clots prepared inside tube I were infused into the lower pulmonary artery through tube II, using gentle syringe pressure (Fig. 1: 8). Local PA was performed to confirm the embolism. Thereafter, the animals were allowed to recover from anesthesia and returned to their regular diet with food and water ad libitum. Oral, entericcoated indomethacin tablets $(0.5 \mathrm{mg} / \mathrm{kg}, 3$ times/day for 3 days) were provided for pain relief, and oral TXA $(110 \mathrm{mg} / \mathrm{kg}$, every $12 \mathrm{~h}$, for the duration of the experiment) was provided to inhibit endogenous fibrinolysis. Prophylactic penicillin $(80,000 \mathrm{U} / \mathrm{kg}$, twice daily for 1 week) was also provided for prevention of infections. 


\section{Euthanasia and thrombopathology}

One or two weeks after embolization, local PA was performed once more and various parameters were recorded. In the reperfusion subgroup, reperfusion (embolectomy) was performed and the animals were mechanically ventilated. Briefly, a right thoracotomy was performed through the fifth intercostal space. The right lower pulmonary lobe was mobilized, after dividing the pulmonary ligament, and the hilar structures were then dissected free. A Fogarty arterial embolectomy was performed as soon as possible, based on the exact location of the thrombus. Thereafter, the lower pulmonary artery was observed for reperfusion changes for $6 \mathrm{~h}$. All animals were euthanized by exsanguination under deep anesthesia. The lungs were removed and the lower pulmonary arteries, with thrombi, were dissected, and fixed in a $10 \%$ aqueous formalin solution.

\section{Investigating the effects and pathological, cellular mechanisms after chronic PTE and LIRI}

A thrombo-pathology study was conducted using histological and paraffin sections stained with hematoxylin and eosin (HE) and phosphotungstic acid-hematoxylin (PTAH). The gross lung appearance were investigated in four groups. The thrombus was shown after embolectomy from the right lower pulmonary lobar artery. The formalin-fixed lung tissues were embedded in paraffin, cut into 4-mmthick tissue slices and were stained with $\mathrm{HE}$ for investigating.

\section{Statistical analysis}

SPSS 11.0 (IBM, Armonk, NY, USA) software was used for the statistical analyses. Numerical parameters with normal Gaussian distribution (according to the Kolmogorov-Smirnov test) were expressed as mean \pm standard deviation $(\bar{x} \pm s)$. Differences in the parameters, among the post-embolization time points within each group or subgroup, were analyzed using repeated measures analysis of variance; a $P$ value of $<0.05$ was considered significant.

\section{Results}

\section{Pulmonary angiography}

Filling defects were not observed in the full or local PAs in the posterior-anterior projection, before embolization (Figs. 1: 7, 2: L1a, 3: R1a). After embolization, the local PA demonstrated an irregular shape of the lower lobar artery with cup-like and cut-off perfusion defects (Figs. 2:
L1b, 3: R1b). After 1 week, PA demonstrated arterial wall irregularities, enlarged proximal parts of the lower artery, and abrupt vessel cut-off perfusion defects, with stria contrast medium filling along the arterial wall, or irregular ramp perfusion defects (Figs. 2: L1c, 3: R1c).

\section{Parameter changes (Table 1)}

\section{Vital signs}

Within each PTE group, the RR and HR increased significantly following clot infusion. After embolization, there were no significant changes in $\mathrm{MBP}$, compared with the baseline $(P>0.05)$, among the groups and subgroups.

\section{Blood gases}

After embolization, the $\mathrm{PaO}_{2} / \mathrm{FiO}_{2}$ decreased significantly after embolization $(P<0.05)$; for example, in the 1 -week subgroup of group RL, the post-embolization value was $390.21 \pm 96.53 \mathrm{mmHg}$ compared to a baseline value of $507.21 \pm 50.21 \mathrm{mmHg}(P<0.05)$. These values increased gradually, toward the baseline value, 1-2 weeks after embolization.

\section{Hemodynamic parameters}

The MPAP and PVR increased as the CO decreased after embolization $(P<0.05)$. For example, in the 1 -week subgroup of group RL, the post-embolization MPAP was $22 \pm 2 \mathrm{mmHg}$, compared to a baseline value of $14 \pm 2 \mathrm{mmHg}(P<0.05)$, and the post-embolization PVR was $555.12 \pm 61.01 \mathrm{dyne} / \mathrm{s} / \mathrm{cm}^{-5}$, compared to a baseline value of $203.11 \pm 38.1 \mathrm{dyne} / \mathrm{s} / \mathrm{cm}^{-5}(P<0.05)$, with a post-embolization $\mathrm{CO}$ of $2.09 \pm 0.35 \mathrm{~L} / \mathrm{min}$, compared to a baseline value of $3.01 \pm 0.29 \mathrm{~L} / \mathrm{min}(P<0.05)$. After 1 week, the MPAP was significantly higher $(20 \pm 3 \mathrm{mmHg})$ than the baseline value $(14 \pm 2 \mathrm{mmHg})$ $(P<0.05)$, whereas the $\mathrm{CO}$ demonstrated a decreasing trend with a difference that was not significant compared to baseline $(P>0.05)$. After 1 or 2 weeks, the PVR increased significantly, compared with the baseline value. For example, the PVR in the 1-week subgroup was $422.01 \pm 52.2 \mathrm{dyne} / \mathrm{s} / \mathrm{cm}^{-5}$, compared with a baseline value of $203.11 \pm 38.1 \mathrm{dyne} / \mathrm{s} / \mathrm{cm}^{-5}(P<0.05)$; in the 2-week subgroup, the PVR was $302.99 \pm 50.02 \mathrm{dyne} / \mathrm{s} /$ $\mathrm{cm}^{-5}$, compared with a baseline value of $183.12 \pm 44.09 \mathrm{dyne} / \mathrm{s} / \mathrm{cm}^{-5}(P<0.05)$. There were no significant changes in the CVP and PAWP $(P>0.05$, data not shown) at different time points among the groups and subgroups. 

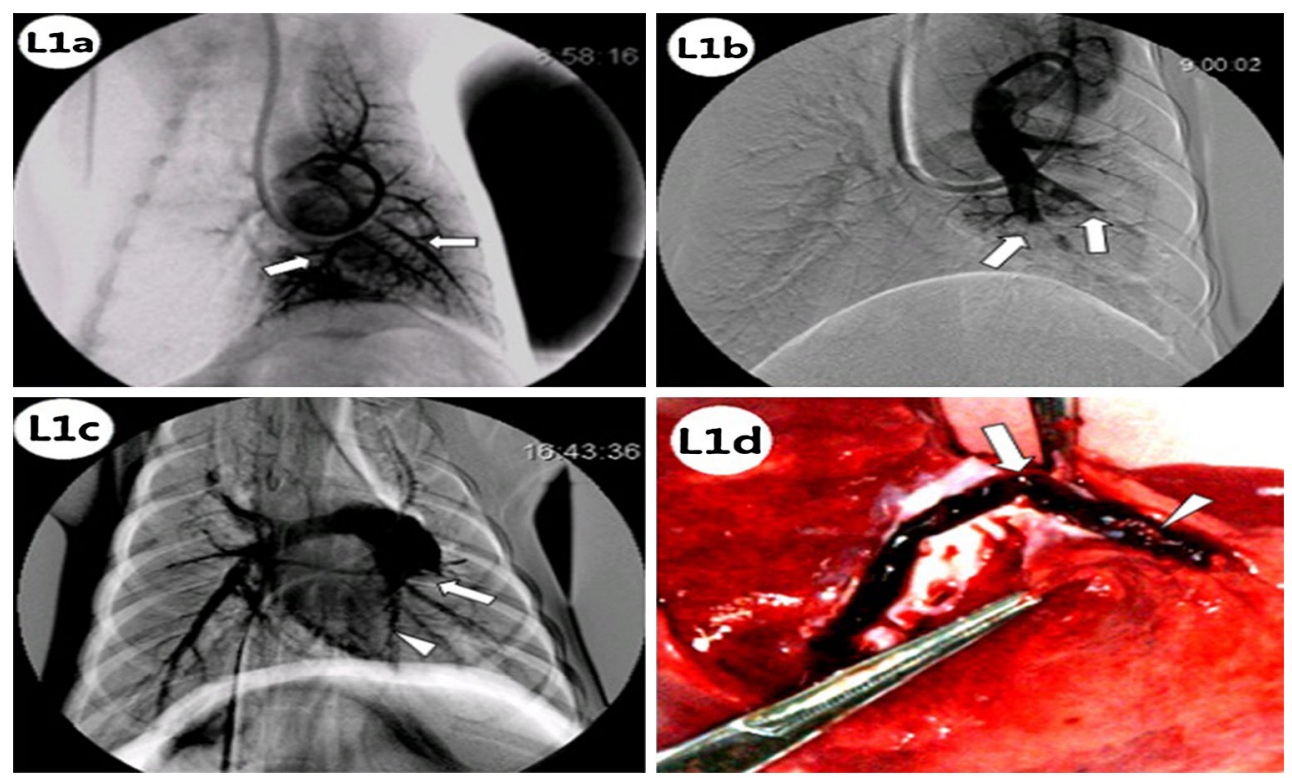

Fig. 2 The pulmonary arteriography (PA) and macroscopic pathology of pulmonary artery in Group LL were showed. L1a In Group LL, normal filling with contrast media (indicated by white arrows) was observed in the left lower lobar artery during local PA, before embolization. L1b After embolization, local PA showed a left lower lobar artery irregular ramp and cut-off perfusion defects (white arrows). L1c After 1 week, PA showed arterial wall irregularities, enlarged proximal sections of left lower pulmonary arteries, and

abrupt vessel cut-off perfusion defects (white arrow) with stria contrast medium filling along the arterial wall (white arrow head). L1d One week after embolization, macroscopic pathology examination indicated bifurcal, reddish-brown thrombi firmly adherent to the pulmonary artery wall (white arrows) along with the presence of multiple, irregular, pink, granulation-like protrusions on the thrombus surface (white arrow head)

\section{Pathological and cellular mechanisms after chronic PTE and LIRI}

\section{Pulmonary artery angiography is closely concordant with pulmonary artery pathology including thrombo- pathology with cellular proliferation}

Pulmonary artery angiography is concordant with the pulmonary artery pathology after the lower pulmonary arteries, with thrombi, were dissected according to the angiography. In the 1-week subgroup of group RL, the pathological examination showed that the organized tissues on the surfaces of the thrombi were pale orange-pink in color with some collagen deposited, and they had invaded the coarse fibrin nests of the thrombus (Fig. 3: R1e). In the 2-week subgroup, there was irregular hyperplasia and hypertrophy of the right lower arterial intima and media (Fig. 4: R2c), irregular vascular walls with neointimal hyperplasia and much more collagen deposition. There may exist an interspace between intima and media (Fig. 4: R2d), and intimal hyperplasia with concentric lamellae (onion-like), composed of some cellular tissue separated by elastic fibers (Fig. 4: R2e). In addition, the pulmonary arterial neointimal hyperplasia was irregularly arranged with multilayer endothelial cells, infiltrated with some inflammatory cells (Fig. 4: R2f). 2 weeks after embolization, in group RL, the

thrombi were organized with multiple recanalization channels (Fig. 5: R3a). The histological sections indicated that the fibrin networks of the thrombi were embedded by neointimal hyperplasia from the pulmonary artery wall with collagen deposited (Fig. 5: R3b).

\section{Pathological and cellular effects of LIRI after embolectomy}

The Sham group showed a normal lung appearance, with pink color (Fig. 6a) and normal intact alveolar structures in the right lower lung (Fig. 6a). The 1-week subgroup had reddish-gray lungs, with some atelectasis (Fig. 6b) and some collapsed alveolar structures (Fig. 6b), as well as a few exudative cells in the alveolar space. The 2-week subgroup had dark red lungs, with more obvious atelectasis (Fig. 6c) and more obviously collapsed alveolar structures with thickened alveolar septa (Fig. 6c). The thrombus was shown after embolectomy from the right lower pulmonary lobar artery (Fig. 6d). The thrombus was a complete, elongated strip with multiple branches consistent with the pulmonary artery branches. The reperfusion subgroup had red lungs that were congested and swollen (Fig. 6e), with destroyed and incomplete alveolar structures as well as a large number of exudative cells (Fig. 6d1), primarily neutrophils, infiltration into the alveolar space and exudate(Fig. 6d2). 

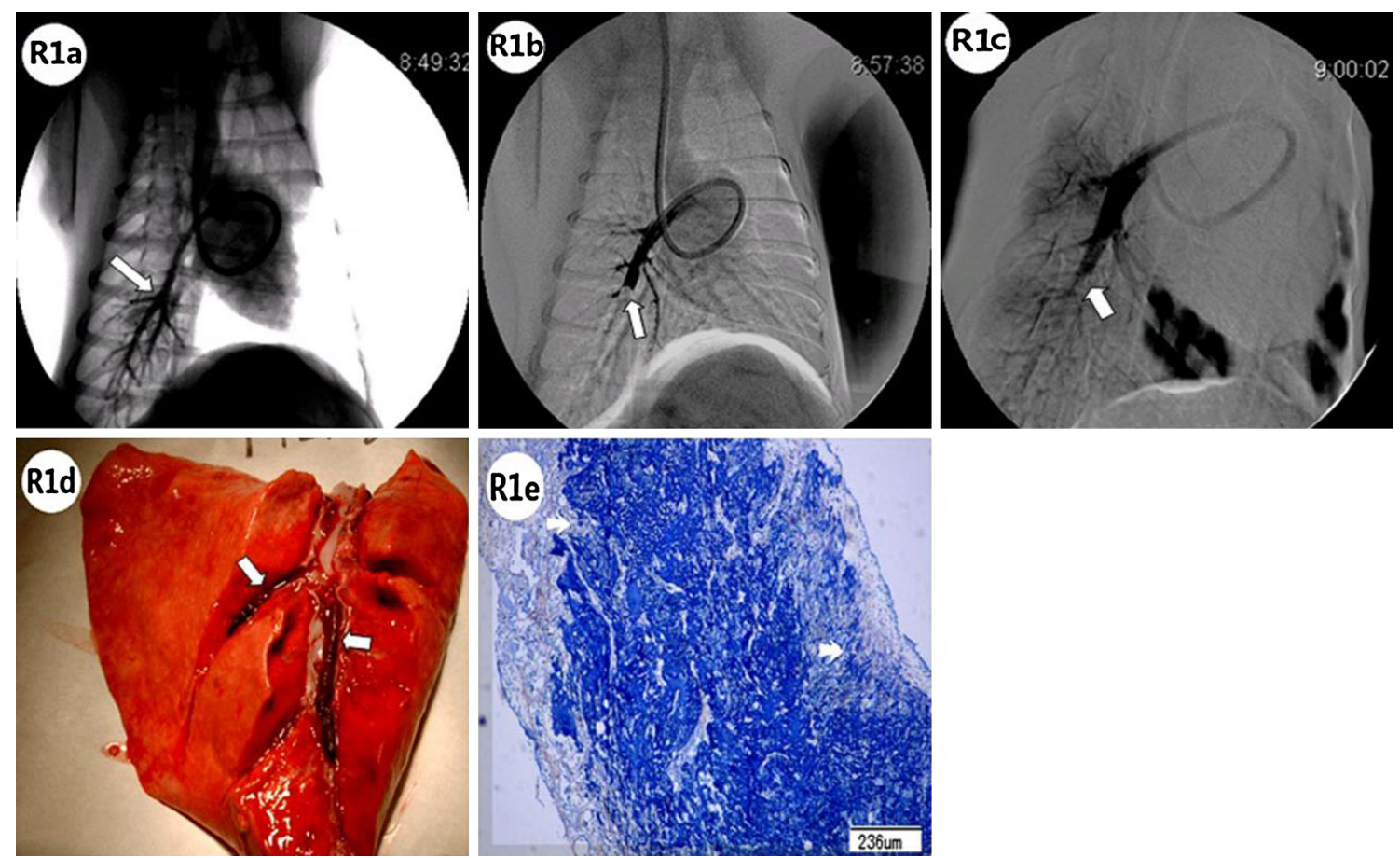

Fig. 3 The pulmonary arteriography (PA), macroscopic pathology and pathology of pulmonary artery in the 1-week subgroup of Group RL were showed. R1a In the 1-week subgroup of Group RL, good filling with contrast media (indicated by white arrows) was observed in the right lower lobar artery on local PA, before embolization. R1b After embolization, PA showed right lower lobar artery cup-like perfusion defects (white arrow). R1c After 1 week, PA showed irregular ramp perfusion defects (white arrow). R1d 1 week after embolization, the macroscopic pathology revealed bifurcal, reddishbrown thrombi, with coarse surfaces, firmly adhered to the right lower lobar artery wall (white arrows). R1e Pathology showed organized tissue on the surfaces of the thrombi, with pale orange-pink covers, and invasive growth (white arrows) into the navy blue coarse fibrin nests of the thrombus (PTAH stain)

\section{Discussion}

\section{A modified chronic PTE model and pulmonary angiography findings}

In most cases, obtaining PTE site samples from human patients is not possible; therefore, animal models are often used for studying the disease.

\section{Major hemodynamic changes in the model}

In our model, we were able to embolize specific lobar arteries with blood clots. In patients with PTE, the lower pulmonary lobar artery is commonly involved due to its extensive circulation [14]; hence, we aimed to embolize these vessels in the currently described model. After embolization, the MPAP and PVR increased, and then gradually reduced as the thrombi resolved, or circulatory reorganization or recanalization occurred. However, even 2 weeks after embolization, the PVR remained significantly higher than the baseline values, mimicking some hemodynamic changes associated with CTEPH.

\section{Pulmonary angiography findings in the model}

PA remains the gold standard modality for diagnosing PTE. During 1 or 2 weeks after embolization, PA demonstrated arterial wall irregularities, enlarged proximal portions of the lower pulmonary artery, abrupt vessel cutoff perfusion defects, stria contrast medium filling along the stiff arterial walls, and abrupt vascular narrowing, which are common manifestations of chronic PTE or CTEPH.

A dog's life span is generally $11-14$ years, which is approximately one-sixth of the human lifespan. Thus, we speculated that the pathophysiological changes occurring within 2 weeks, in a dog, may somewhat reflect the changes observed over 12 weeks in humans, which is the chronic PTE or CTEPH threshold time [15-17]. Chronic PTE are usually recognized as being synonymous with CTEPH. However, serial pulmonary angiographic and lung scan studies have revealed that approximately $15-25 \%$ of acute PTE patients show only partial resolution of their pulmonary vascular obstructions in follow-up lung scans, performed 3-4 months after the primary embolic event [18-20]. Over a period of months or years, approximately 
Table 1 Changes in vital signs, blood gases, and hemodynamic parameters among the subgroup $(\bar{x} \pm \mathrm{s})$

\begin{tabular}{|c|c|c|c|c|c|}
\hline Parameters & Group & Subgroup (each $\mathrm{n}=10$ ) & Before embolization & After embolization & After 1 or 2 weeks \\
\hline RR & LL & 1-week & $23 \pm 2$ & $34 \pm 2^{*}$ & $27 \pm 3^{* \#}$ \\
\hline \multirow[t]{3}{*}{ Times/min } & \multirow[t]{3}{*}{ RL } & Sham & $24 \pm 3$ & $25 \pm 3$ & $24 \pm 2$ \\
\hline & & 1-week & $23 \pm 2$ & $35 \pm 3^{*}$ & $27 \pm 2^{* \#}$ \\
\hline & & 2-week & $24 \pm 2$ & $34 \pm 3^{*}$ & $27 \pm 3^{* \#}$ \\
\hline HR & LL & 1-week & $144 \pm 5$ & $185 \pm 6^{*}$ & $151 \pm 5^{* \#}$ \\
\hline \multirow[t]{3}{*}{ Beats/min } & \multirow[t]{3}{*}{ RL } & Sham & $151 \pm 5$ & $150 \pm 8$ & $149 \pm 7$ \\
\hline & & 1-week & $143 \pm 6$ & $185 \pm 5^{*}$ & $151 \pm 6^{* \#}$ \\
\hline & & 2-week & $152 \pm 6$ & $181 \pm 7^{*}$ & $156 \pm 9^{* \#}$ \\
\hline \multirow[t]{4}{*}{ MBP (mmHg) } & LL & 1-week & $108 \pm 16$ & $103 \pm 17$ & $102 \pm 14$ \\
\hline & \multirow[t]{3}{*}{$\mathrm{RL}$} & Sham & $95 \pm 6$ & $96 \pm 7$ & $94 \pm 11$ \\
\hline & & 1-week & $108 \pm 18$ & $103 \pm 19$ & $102 \pm 14$ \\
\hline & & 2-week & $110 \pm 17$ & $109 \pm 15$ & $109 \pm 13$ \\
\hline \multirow[t]{4}{*}{$\mathrm{pH}$} & LL & 1-week & $7.32 \pm 0.11$ & $7.28 \pm 0.10$ & $7.29 \pm 0.10$ \\
\hline & \multirow[t]{3}{*}{$\mathrm{RL}$} & Sham & $7.35 \pm 0.05$ & $7.34 \pm 0.02$ & $7.32 \pm 0.06$ \\
\hline & & 1-week & $7.30 \pm 0.09$ & $7.28 \pm 0.11$ & $7.28 \pm 0.09$ \\
\hline & & 2-week & $7.32 \pm 0.04$ & $7.32 \pm 0.06$ & $7.28 \pm 0.07$ \\
\hline \multirow[t]{4}{*}{$\mathrm{PaO}_{2} / \mathrm{FiO}_{2}(\mathrm{mmHg})$} & LL & 1-week & $505.21 \pm 56.35$ & $390.21 \pm 96.53^{*}$ & $497.89 \pm 94.23^{\#}$ \\
\hline & \multirow[t]{3}{*}{ RL } & Sham & $492.89 \pm 31.43$ & $488.33 \pm 15.35$ & $510.52 \pm 41.22$ \\
\hline & & 1-week & $507.21 \pm 50.21$ & $390.21 \pm 93.56^{*}$ & $496.99 \pm 91.73^{\#}$ \\
\hline & & 2-week & $492.44 \pm 48.56$ & $375.88 \pm 88.87^{*}$ & $467.68 \pm 53.21^{\#}$ \\
\hline \multirow[t]{4}{*}{$\mathrm{PaCO}_{2}(\mathrm{mmHg})$} & LL & 1-week & $38.15 \pm 9.69$ & $36.88 \pm 5.9$ & $436.69 \pm 7.79$ \\
\hline & \multirow[t]{3}{*}{ RL } & Sham & $38.56 \pm 3.59$ & $37.33 \pm 6.8$ & $336.51 \pm 6.88$ \\
\hline & & 1-week & $38.66 \pm 6.01$ & $37.52 \pm 5.1$ & $436.66 \pm 6.32$ \\
\hline & & 2-week & $36.23 \pm 3.99$ & $34.44 \pm 3.7$ & $336.89 \pm 6.01$ \\
\hline \multirow[t]{4}{*}{ MPAP (mmHg) } & LL & 1-week & $15 \pm 2$ & $21 \pm 3^{*}$ & $20 \pm 3^{*}$ \\
\hline & \multirow[t]{3}{*}{ RL } & Sham & $14 \pm 2$ & $15 \pm 2$ & $14 \pm 2$ \\
\hline & & 1-week & $14 \pm 2$ & $22 \pm 2^{*}$ & $20 \pm 3^{*}$ \\
\hline & & 2-week & $14 \pm 2$ & $22 \pm 3^{*}$ & $17 \pm 2^{\#}$ \\
\hline \multirow[t]{4}{*}{$\mathrm{CO}(\mathrm{L} / \mathrm{min})$} & LL & 1-week & $3.13 \pm 0.32$ & $2.28 \pm 0.25^{*}$ & $2.89 \pm 0.33$ \\
\hline & \multirow[t]{3}{*}{ RL } & Sham & $3.02 \pm 0.29$ & $2.93 \pm 0.29$ & $2.92 \pm 0.29$ \\
\hline & & 1-week & $3.01 \pm 0.29$ & $2.09 \pm 0.35^{*}$ & $2.60 \pm 0.29$ \\
\hline & & 2-week & $3.19 \pm 0.28$ & $2.19 \pm 0.39^{*}$ & $3.16 \pm 038^{\#}$ \\
\hline \multirow[t]{4}{*}{ PVR (dyne/s/cm ${ }^{-5}$ ) } & LL & 1-week & $217.33 \pm 38.9$ & $548.20 \pm 66.10^{*}$ & $419.18 \pm 56.2^{*}$ \\
\hline & \multirow[t]{3}{*}{ RL } & Sham & $211.0 \pm 35.55$ & $220.29 \pm 41.22$ & $189.21 \pm 42.22$ \\
\hline & & 1-week & $203.11 \pm 38.1$ & $555.12 \pm 61.01^{*}$ & $422.01 \pm 52.2^{*}$ \\
\hline & & 2-week & $183.12 \pm 44.09$ & $401.22 \pm 71.11^{*}$ & $302.99 \pm 50.02^{*}$ \\
\hline
\end{tabular}

$R R$ respiration rate, $H R$ heart rate, $M B P$ mean blood pressure, $M P A P$ mean pulmonary artery pressure, $\mathrm{PaO}_{2}$ oxygen partial pressure, $\mathrm{FiO}_{2}$ fraction of inspired oxygen, $\mathrm{PaCO}_{2}$ carbon dioxide partial pressure, $\mathrm{CO}$ cardiac output, $\mathrm{PVR}$ pulmonary vascular resistance

* $P<0.05$ for the comparison with parameters recorded before embolization. ${ }^{\#} P<0.05$ for the comparison with parameters recorded after embolization

$3 \%$ of patients with acute PTE develop CTEPH [21]. The underlying pathophysiological mechanisms are largely unknown [22] [23]. Many studies have shown that some patients demonstrate "chronic pulmonary embolism" features, without evidence of chronic pulmonary hypertension, however, they may develop pulmonary hypertension [2426]. Thus, this new insight has added additional caveats about the natural history of acute pulmonary embolisms that should be considered [22] [27]. In our study, 2 weeks after embolization, the appearance of webs or bands and abrupt vascular narrowing and intimal irregularities was consistent with the pulmonary angiography findings of CTEPH cases [28-30] and may represent a multiply recanalized thrombus [31]. 

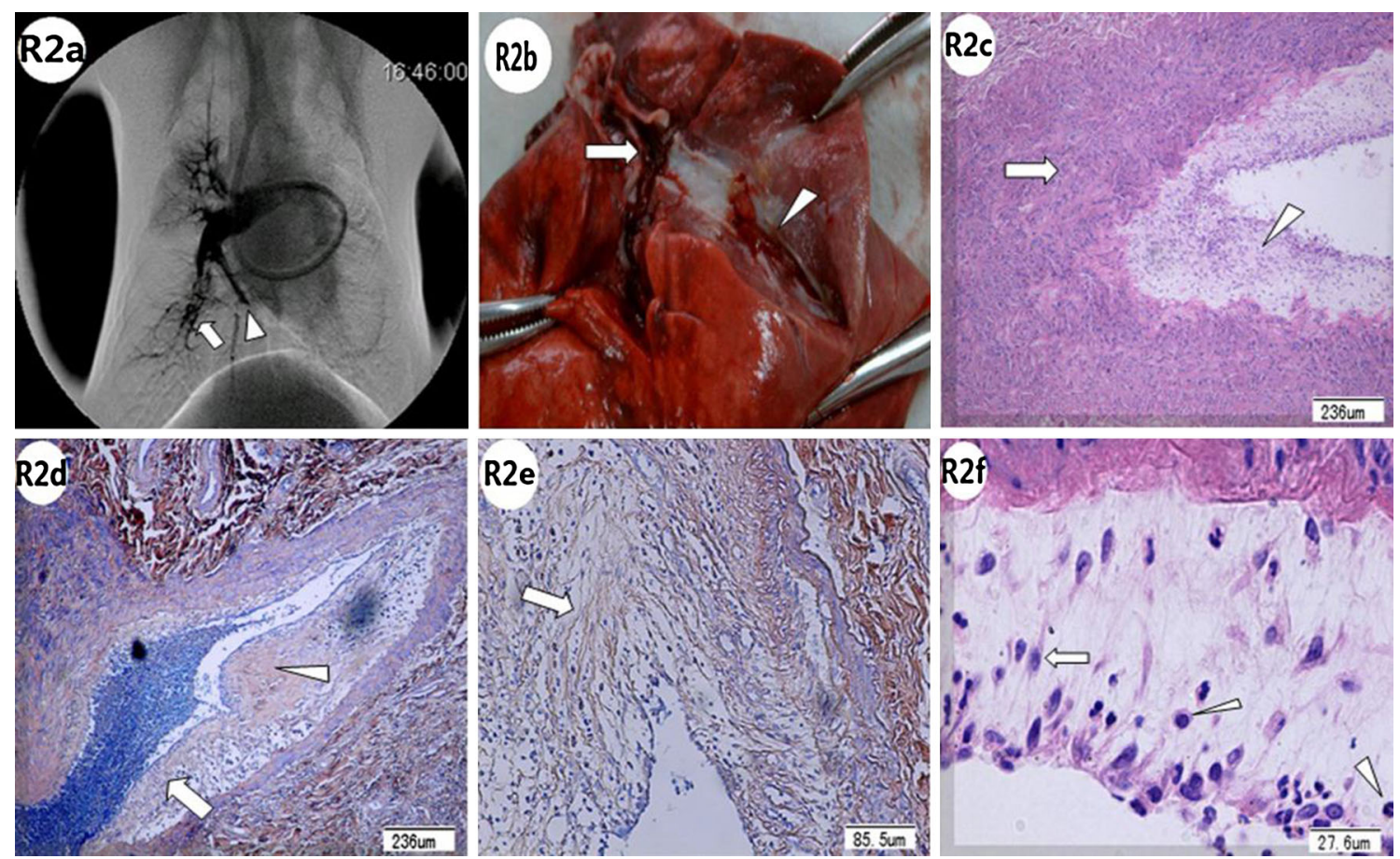

Fig. 4 The pulmonary arteriography (PA), macroscopic pathology and pathology of pulmonary artery in the 2-week subgroup of Group RL were showed. R2a 2 weeks after embolization in Group RL, local PA showed arterial wall irregularities in addition to webs or bands (indicated by white arrow), stria contrast medium filling along the arterial wall, and abrupt vascular narrowing (white arrow head). R2b Pathology examination revealed a constricted thrombus with an irregular surface, reddish-brown proximal region (white arrow), and pink distal region (white arrow head) that was adhered firmly to the pulmonary artery wall. R2c Pathology examination showed irregular hyperplasia and hypertrophy of the right lower lobar artery intima (white arrow head) and media (white arrow) (Hematoxylin and Eosin stain). R2d Pathology examination showed irregular vascular walls with neointimal hyperplasia (white arrow) and collagen deposition (white arrow head) (PTAH stain). There existed a major interspace between intima and media (black arrow head). R2e Intimal hyperplasia with concentric lamellar (onion-like) structures, composed of some cellular tissue, was separated by elastic fibers (white arrow) (PTAH stain). R2f Pulmonary arterial neointimal hyperplasia was irregularly arranged with multilayered endothelial cells (white arrow) and some inflammatory cell infiltration (white arrow heads) (Hematoxylin and Eosin stain)

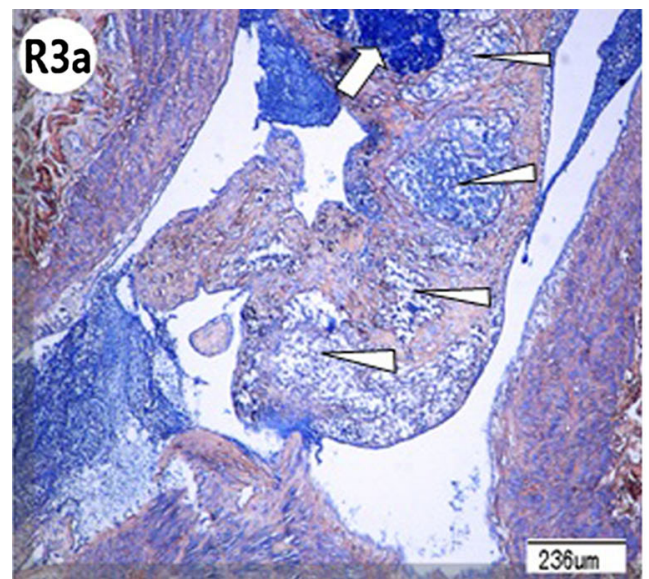

Fig. 5 The pathology of thrombi in Group RL was showed 2 weeks after embolization. R3a 2 weeks after embolization in Group RL, the thrombi were organized (indicated by white arrow) with multiple recanalization channels (white arrow heads) (PTAH stain). R3b

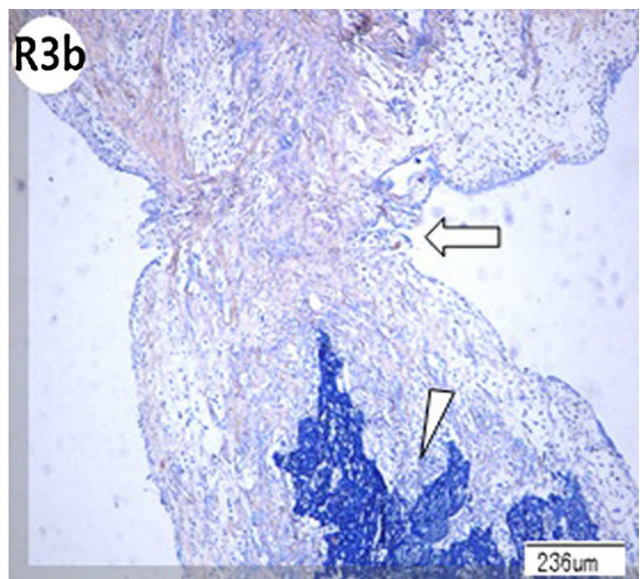

Histological sections indicated that the fibrin networks of the thrombi were invaded (white arrow head) by neointimal hyperplasia and collagen from the pulmonary arterial wall (white arrow) (PTAH stain) 

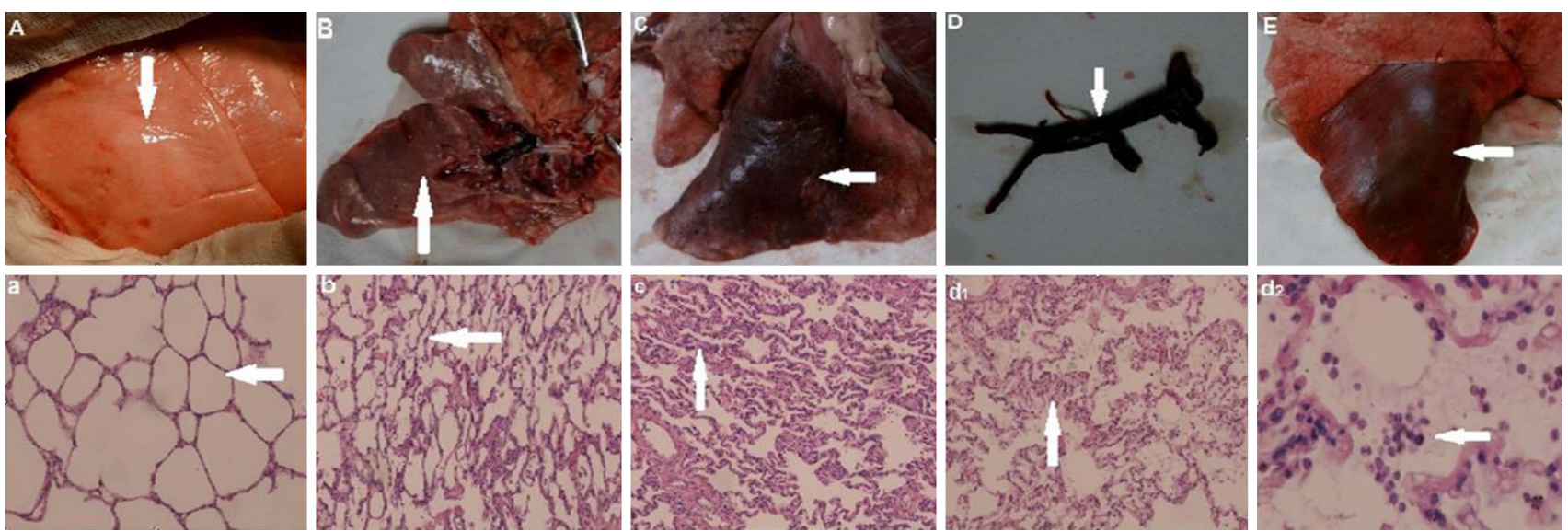

Fig. 6 The macroscopic pathology and pathology of pulmonary artery among different groups were showed. The Sham group showed a normal gross lung (A, white arrow) and normal right lower lung (a, Hematoxylin and Eosin stain, $10 \times$, white arrow). The 1-week subgroup had reddish-gray lungs, with some atelectasis (B, white arrow) and some collapsed alveolar structures (b. Hematoxylin and Eosin stain, $10 \times$, white arrow), as well as a few exudative cells in the alveolar space. The 2-week subgroup had dark red lungs, with more obvious atelectasis $(\mathbf{C}$, white arrow) and more obviously collapsed alveolar structures with thickened alveolar septa (c, Hematoxylin and
Eosin stain, $10 \times$, white arrow). The thrombus is shown after embolectomy from the right lower pulmonary lobar artery (white arrow). The thrombus was a complete, elongated strip with multiple branches consistent with the pulmonary artery branches. The reperfusion subgroup had red lungs that were congested and swollen (E, white arrow), with destroyed and incomplete alveolar structures as well as a large number of exudative cells $(d 1$, Hematoxylin and Eosin stain, $10 \times$, white arrow), mainly neutrophils ( $d 2$, Hematoxylin and Eosin stain, $40 \times$, white arrow), and exudate

fibrinolytic activity and the number of recanalized lesions correlates with the reduction in PVR [31].

\section{Cellular and molecular mechanisms after chronic PTE in the model}

In the 2-week subgroup in our model, many findings demonstrated the cellular and molecular mechanisms after chronic PTE. These findings included the concentric, lamellar (onion-like) intimal hyperplasia, with fibrous septa; multilayered, irregular arrangements of endothelial cells; and the infiltration of inflammatory cells. These findings can also be reflected by the histological examinations of endarterectomized tissues from patients with CTEPH [14, 32, 33]. The microenvironment provided by the unresolved clot and inflammatory cells may stimulate erroneous cell proliferation, promote the endothelial-mesenchymal transition, cause endothelial injury and/or induce endothelial cell (EC) dysfunction [33, 34]; furthermore, the infiltration of inflammatory cells, such as leukocytes or monocytes, into the vascular wall may release proinflammatory cytokine macrophage chemoattractant protein-1 (MCP-1) and interleukins 1 and 6, which can potentially act as chemoattractants for fibroblasts or smooth muscle cells [15]. Because of ischemia, some collapsed alveolar structures, thickened alveolar septa, and collagen fibers stained blue and a few exudative cells, in the alveolar space were also demonstrated in our recent study [35]. All these 
are critical for future investigations into the of the disease and for the development of novel and therapeutic approaches.

\section{The mechanisms of LIRI after embolectomy-mediated reperfusion}

According to our experimental model, precise embolization into the intended location also facilitates the use for preclinical investigations into interventional management or open pulmonary thromboembolectomy as clinical practice $[36,37]$. In our model, we investigated LIRI after performing the embolectomy to study the exact location of the thrombus and related effects. The experimental and clinical observations suggest that the main dysfunctional characteristic of LIRI is an increase in pulmonary microvascular permeability, and that the transendothelial migration of inflammatory cells may be a critical step in the development of dysfunction after LTX treatment, and may be a source of inflammatory mediators [38]. In our study, the incomplete and destroyed alveolar structures, in conjunction with large numbers of exudative cells, mainly neutrophils, and exudation distal to the clot, after embolectomy in the reperfusion subgroup following 2 weeks of ischemia, provided strong experimental evidence of similar mechanisms for LIRI in PTE and lung transplantation. In addition, the methods and procedures used in our model can also be used to selectively embolize other target organs or blood vessels, such as certain brain vessels, with the guidance of a Swan-Ganz float catheter.

\section{Limitations and clinical implications}

Although the process of chronic PTE was mimicked in our research, some distinctions remain between our model and the actual process. Blood clots induced in vitro or ex vivo are distinct from the laminar and heterogeneous venous thrombi formed within a deep vein [39]. An ideal "PTE animal model" does not exist because animals do not develop spontaneous DVTs [2].

Pulmonary vascular wall remodeling and some cellular and molecular mechanisms after chronic PTE are demonstrated in our model. The possible interspace between proliferative intima and media in our model may prove the possibility for thrombarterectomy, as successfully performed in the patients with CTEPH in clinical pracitce. After reperfusion, the incomplete and destroyed alveolar structure, and cells mainly neutrophils may play an important role in the LIRI in the model, implying antiinflammation to relieve the LIRI after thrombarterectomy in clinical CTEPH cases. However, there exist difference with the biological processes such as thrombus organiztion between the dogs and human beings. Moreover, the mechanisms of LIRI are profound and may include neutrophil activation, cytokines, ROS, arachidonic acid derivatives, complement, etc., causing cellular damage. Therefore, further studies shoud focus intensively on the interactions among inflammatory response factors during LIRI in PTE. We believe that progress will be made towards an improved understanding of PTEs, including CTEPH, chronic PTE, and LIRI after reperfusion in clinical practice.

A modified canine PTE model can be established by selectively introducing blood clots into specific pulmonary lobar arteries using Swan-Ganz catheters under fluoroscopic guidance. This model with the similar pulmonary angiography findings may mimic the clinical chronic PTE cases. The pathological and cellular mechanisms related to chronic PTE mainly involve the recanalization of thrombi and the remodelling of pulmonary artery with the concentric, lamellar (onion-like) intimal hyperplasia,multilayered, irregular arrangements of endothelial cells, and the infiltration of inflammatory cells. On the other hand, the mechanisms related to the LIRI after embolectomy-mediated reperfusion involve the destroyed, incomplete alveolar structures, and infiltration of inflammatory cells, primarily neutrophils and the detailed mechanisms warrant further investigation.

Acknowledgments This research was supported by Programs of National Natural Science Foundation of China (81570264), Young and Middle-Aged Talent Cultivation of Fujian Provincial Health System (2015-ZQN-ZD-18) and Fujian Provincial Medical Innovation Subject (2014-CXB-12). The authors declare that they do not have any competing or financial interests.

Open Access This article is distributed under the terms of the Creative Commons Attribution 4.0 International License (http://creati vecommons.org/licenses/by/4.0/), which permits unrestricted use, distribution, and reproduction in any medium, provided you give appropriate credit to the original author(s) and the source, provide a link to the Creative Commons license, and indicate if changes were made.

\section{References}

1. Gibbs H, Fletcher J, Blombery P et al (2011) Venous thromboembolism prophylaxis guideline implementation is improved by nurse directed feedback and audit. Thromb J 9:7

2. Diaz JA, Obi AT, Myers DD Jr et al (2012) Critical review of mouse models of venous thrombosis. Arterioscler Thromb Vasc Biol 32:556-562

3. Kjaergaard B, Kristensen SR, Risom M et al (2009) A porcine model of massive, totally occlusive, pulmonary embolism. Thromb Res 124:226-229

4. Runyon MS, Gellar MA, Sanapareddy N et al (2010) Development and comparison of a minimally-invasive model of autologous clot pulmonary embolism in Sprague-Dawley and Copenhagen rats. Thromb J 8:3

5. Marsh JJ, Konopka RG, Lang IM et al (1994) Sppression of thrombolysis in a canine model of pulmonary embolism. Circulation 90:3091-3097 
6. Iskandar SB, Shabaneh B, Brahmbhatt VR et al (2004) Rightsided heart thromboembolism and pulmonary embolism. Tenn Med 97:34-36

7. Yamada E, Zhang Y, Davies R et al (2002) Phased-array intracardiac echocardiographic imaging of acute cardiovascular emergencies: experimental studies in dogs. J Am Soc Echocardiogr 15:1309-1314

8. Roehl AB, Steendijk P, Baumert JH et al (2009) Comparison of 3 methods to induce acute pulmonary hypertension in pigs. Comp Med 59:280-286

9. Weissmann N, Sydykov A, Kalwa $\mathrm{H}$ et al (2012) Activation of TRPC6 channels is essential for lung ischaemia-reperfusion induced oedema in mice. Nat Commun 3:649

10. Raikhelkar JK, Milla F, Darrow B et al (2011) Adjuvant therapy with methylene blue in the treatment of right ventricular failure after pulmonary embolectomy. Heart Lung Circ 20:234-236

11. Shearin AL, Ostrander EA (2010) Leading the way: canine models of genomics and disease. Dis Model Mech 3:27-34

12. Klocking HP (1978) Pharmacology of streptokinase. In: Markwardt $F$ (ed) Fibrinolytics and antifibrinolytics. Springer, New York, pp 151-177

13. Virchow R (1846) Thrombosis and emboli. Science History Publications, Canton

14. Wagenvoort CA (1995) Pathology of pulmonary thromboembolism. Chest 107:10S-17S

15. Lang I (2010) Advances in understanding the pathogenesis of chronic thromboembolic pulmonary hypertension. Br J Haematol 149:478-483

16. Mehta S, Helmersen D, Provencher S et al (2010) Diagnostic evaluation and management of chronic thromboembolic pulmonary hypertension: a clinical practice guideline. Can Respir J $17: 301-334$

17. Tapson VF, Humbert M (2006) Incidence and prevalence of chronic thromboembolic pulmonary hypertension. Proc Am Thorac Soc 3:564-567

18. Tow DE, Wagner HN Jr (1967) Recovery of pulmonary arterial blood flow in patients with pulmonary embolism. N Engl J Med 276:1053-1059

19. The Urokinase Pulmonary Embolism Trial Study Group (1973) The urokinase pulmonary embolism trial: a national cooperative study. Circulation 47(Suppl 2):II1-108

20. Wartski M, Collignon MA (2000) Incomplete recovery of lung perfusion after 3 months in patients with acute pulmonary embolism treated with antithrombotic agents. J Nucl Med 41:1043-1048

21. Lang IM, Klepetko W (2008) Chronic thromboembolic pulmonary hypertension: an updated review. Curr Opin Cardiol 23:555-559

22. Peterson KL (1999) Acute pulmonary embolism. Has its evolution been redefined. Circulation 99:1280-1283

23. Klok FA, Mos IC, van Kralingen KW (2012) Chronic pulmonary embolism and pulmonary hypertension. Semin Respir Crit Care Med 33:199-204
24. Meyer G, Planquette B, Sanchez O (2008) Long-term outcome of pulmonary embolism. Curr Opin Hematol 15:499-503

25. Zhang Y, Zhang Y, Zhang J (2009) A diagnostic dilemma of syncope: a patient with chronic pulmonary embolism. BMJ Case Rep. doi:10.1136/bcr.06.2008.0277

26. Douma RA, Oduber CE, Gerdes VE et al (2012) Chronic pulmonary embolism in Klippel-Trenaunay syndrome. J Am Acad Dermatol 66:71-77

27. Miwa S, Shirai M, Kobayashi S et al (2004) Chronic pulmonary thromboembolism pathologically showing homogeneous cellular alveolitis. Intern Med 50:2195-2200

28. Pengo V, Lensing AW, Prins MH et al (2004) Incidence of chronic thromboembolic pulmonary hypertension after pulmonary embolism. N Engl J Med 350:2257-2264

29. Dartevelle P, Fadel E, Mussot S et al (2004) Chronic thromboembolic pulmonary hypertension. Eur Respir J 23:637-648

30. Ley S, Ley-Zaporozhan J, Pitton MB et al (2012) Diagnostic performance of state-of-the-art imaging techniques for morphological assessment of vascular abnormalities in patients with chronic thromboembolic pulmonary hypertension (CTEPH). Eur Radiol 22:607-616

31. Hosokawa K, Ishibashi-Ueda H, Kishi $\mathrm{T}$ et al (2012) Histopathological multiple recannalized lesion is critical element of outcome after pulmonary thromboendarteretomy. Int Heart $\mathrm{J}$ $52: 377-381$

32. Quarck R, Wynants M, Ronisz A et al (2012) Characterization of proximal pulmonary arterial cells from chronic thromboembolic pulmonary hypertension patients. Respir Res 13:27-37

33. Sakao S, Hao H, Tanabe N et al (2012) Endothelial-like cells in chronic thromboembolic pulmonary hypertension: crosstalk with myofibroblast-like cells. Respir Res 12:109-124

34. Maruoka M, Sakao S, Kantake M et al (2012) Characterization of myofibroblasts in chronic thromboembolic pulmonary hypertension. Int J Cardiol 159:119-127

35. Deng CS, Yang MX, Lin QC et al (2014) Beneficial effects of inhaled NO on apoptotic pneumocytes in pulmonary thromboembolism model. Theor Biol Med Model. doi:10.1186/17424682-11-36

36. Lee S, Song SW, Yi G et al (2008) Open pulmonary thromboembolectomy in patients with major pulmonary thromboembolism. Yonsei Med J 49:973-977

37. Yi I, Park JC, Cho KS et al (2011) Pulmonary thromboembolectomy for acute pulmonary thromboembolism. Korean J Thorac Cardiovasc Surg 44:343-347

38. Kohno M, Watanabe M, Goto T et al (2014) Attenuation of lung ischemia-reperfusion injury by rho-associated kinase inhibition in a rat model of lung transplantation. Ann Thorac Cardiovasc Surg 20:359-364

39. Evans CE, Humphries J, Saha P et al (2012) Opinions on mouse models of thrombosis. Thromb Res 130:285-286 